\title{
AnAlysis OF THE $X(1576)$ AS A TETRAQUARK STATE WITH THE QCD SUM RULES
}

\author{
Zhi-Gang Wang ${ }^{1}{ }^{1}$, Shao-Long Wan ${ }^{2}$ \\ ${ }^{1}$ Department of Physics, North China Electric Power University, Baoding 071003, \\ P. R. China \\ ${ }^{2}$ Department of Modern Physics, University of Science and Technology of China, \\ Hefei 230026, P. R. China
}

\begin{abstract}
In this letter, we take the point of view that the $X(1576)$ be tetraquark state which consists of a scalar-diquark and an anti-scalar-diquark in relative $P$-wave, and calculate its mass in the framework of the QCD sum rules approach. The numerical value of the mass $m_{X}=(1.66 \pm 0.14) G e V$ is consistent with the experimental data, there may be some tetraquark component in the vector meson $X(1576)$.
\end{abstract}

PACS numbers: 12.38.Lg; 12.39.Mk

Key Words: tetraquark, QCD sum rules

The BES collaboration observed a broad resonance $X(1576)$ in the $K^{+} K^{-}$invariant mass spectrum in the decay $J / \psi \rightarrow K^{+} K^{-} \pi^{0}$, the pole position is $1576_{-55-91}^{+49+98}$ $i 409_{-12-67}^{+11+32} \mathrm{MeV}$ and the $J^{P C}$ is $1^{--}$[1]. The resonance $X(1576)$ is an isovector due to the $\pi$ produced from the isoscalar $J / \psi$ [2, 3, 4]. The broad resonance cannot be interpreted as any known mesons or their mixing states, it may be a tetraquark state or a meson-meson molecular state, in this letter, we study the possibility of the tetraquark state assignment. In the tetraquark scenario, the $X(1576)$ consists of the basic constituents, the scalar diquarks $[u s],[d s],[\bar{u} \bar{s}]$ and $[\bar{d} \bar{s}]$, the decay $X(1576) \rightarrow K^{+} K^{-}$occurs through the fall apart mechanism with re-arrangement in the color space, the width of the $X(1576)$ can be much larger than the width of any known two-quark vector mesons [2, 3, 4.

It is obvious that the vector tetraquark state has many novel features which are of phenomenological importance and have many observable consequence, one can consult Refs. 2, 3, 4, for details. In this letter, we focus on its substructure and mass, and take the point of view that the vector meson $X(1576)$ with the isospin $I=1$ be a tetraquark state, calculate the value of its mass in the framework of the QCD sum rules approach [5].

In the following, we write down the interpolating current for the vector meson $X(1576)$,

$$
\begin{aligned}
J_{\mu}(x)= & D^{a}(x) \partial_{\mu} \bar{D}^{a}(x)-\partial_{\mu} D^{a}(x) \bar{D}^{a}(x) \\
& -U^{a}(x) \partial_{\mu} \bar{U}^{a}(x)+\partial_{\mu} U^{a}(x) \bar{U}^{a}(x), \\
U^{a}(x)= & \epsilon^{a b c} d_{b}^{T}(x) C \gamma_{5} s_{c}(x), \\
D^{a}(x)= & \epsilon^{a b c} u_{b}^{T}(x) C \gamma_{5} s_{c}(x),
\end{aligned}
$$

\footnotetext{
${ }^{1}$ Corresponding author; E-mail,wangzgyiti@yahoo.com.cn.
} 
where the $a, b, c$ are color indices and the $C$ is the charge conjugation matrix. The constituents $U^{a}(x)$ and $D^{a}(x)$ represent the scalar diquarks $\left(S^{a}\right)$ with the $J^{P}=0^{+}$, they belong to the antitriplet $\overline{3}_{c}$ representation of the color $S U(3)$ group and can cluster together to form the scalar-scalar $\left(S^{a}-\bar{S}^{a}\right)$ type diquark pairs to give the correct spin and parity of the vector mesons $J^{P}=1^{-}$. The attractive interaction of one-gluon exchange favors the formation of diquarks in the color antitriplet $\overline{3}_{c}$, flavor antitriplet $\overline{3}_{f}$ and spin singlet $1_{s}$. The instanton induced force results in strong attraction in the scalar diquark channels and strong repulsion in the pseudoscalar diquark $\left(P^{a}\right)$ channels, we prefer the $S^{a}-\bar{S}^{a}$ type interpolating currents to the $P^{a}-\bar{P}^{a}$ type interpolating currents if the instantons manifest themselves [6, 7]. The strong attractions between the scalar diquark states $S^{a}$ and $\bar{S}^{a}$ in $S$-wave may result in a nonet manifested below $1 G e V$, while the conventional ${ }^{3} P_{0} \bar{q} q$ nonet would have masses about $1.2-1.6 \mathrm{GeV}$ [7, 8]. The additional $P$-wave between the $S^{a}$ and $\bar{S}^{a}$ can lead to the negative parity and much higher mass than $1 \mathrm{GeV}$, for example, the contribution from the $P$-wave is about $480 \mathrm{MeV}$ in the Isgur-Karl model 9, we introduce the relative $P$-wave through the $\partial_{\mu}$ between the $S^{a}$ and $\bar{S}^{a}$. If we take the scalar diquark mass $m_{D}=0.7 \mathrm{GeV}$ from lattice QCD as the input parameter [10], the mass of vector tetraquark is about $2 m_{D}+0.48=1.88 \mathrm{GeV}$, our numerical result $m_{X}=(1.66 \pm 0.14) \mathrm{GeV}$ confirms this naive analysis. In fact, there are large uncertainties about the masses of the colored diquarks, for more literatures and detailed discussions about this subject, one can consult Ref. 11.

In this letter, we study the mass of the $X(1576)$ with the following two-point correlation function,

$$
\Pi_{\mu \nu}(p)=i \int d^{4} x e^{i p \cdot x}\left\langle 0\left|T\left\{J_{\mu}(x) J_{\nu}^{\dagger}(0)\right\}\right| 0\right\rangle .
$$

The correlation function $\Pi_{\mu \nu}(p)$ can be decomposed as

$$
\Pi_{\mu \nu}=-\Pi\left\{g_{\mu \nu}-\frac{p_{\mu} p_{\nu}}{p^{2}}\right\}+\Pi_{0} \frac{p_{\mu} p_{\nu}}{p^{2}}
$$

due to the Lorentz covariance. The transverse part $\Pi$ comes from the contributions of the vector mesons and the longitudinal part $\Pi_{0}$ comes from the scalar mesons. We derive the sum rules with the tensor structure $\left\{g_{\mu \nu}-\frac{p_{\mu} p_{\nu}}{p^{2}}\right\}$, for the scalar meson, one can use the scalar interpolating currents as the ideal choice rather than the vector current $J_{\mu}(x)$ [7].

According to the basic assumption of the current-hadron duality in the QCD sum rules approach [5], we insert a complete series of intermediate states satisfying the unitarity principle with the same quantum numbers as the current operator $J_{\mu}(x)$ into the correlation function in Eq.(2) to obtain the hadronic representation. Isolating the ground state contribution from the pole term of the vector tetraquark state, we obtain the result,

$$
\Pi(p)=\frac{2 f_{X}^{2}}{m_{X}^{2}-p^{2}}+\cdots
$$


where the following definition has been used,

$$
\left\langle 0\left|J_{\mu}(0)\right| X\right\rangle=\sqrt{2} f_{X} \epsilon_{\mu}
$$

the $\epsilon_{\mu}$ is the polarization vector of the $X(1576)$. We have not shown the contributions from the higher resonances and continuum states explicitly for simplicity.

The calculation of the operator product expansion in the deep Euclidean spacetime region is straightforward and tedious, technical details are neglected for simplicity. In this letter, we consider the terms of the vacuum condensates add up to dimension 11, neglect the terms proportional to $m_{s}^{2},\left\langle\bar{q} g_{s} \sigma G q\right\rangle^{2},\left\langle\bar{s} g_{s} \sigma G s\right\rangle^{2}$ and $\left\langle\bar{q} g_{s} \sigma G q\right\rangle\left\langle\bar{s} g_{s} \sigma G s\right\rangle$. In calculation, we take the assumption of the vacuum saturation for the high dimension vacuum condensates, they are always factorized to lower condensates with the vacuum saturation in the QCD sum rules approach, the factorization works well in the large $N_{c}$ limit. Once the analytical result is obtained, then we can take the current-hadron duality below the threshold $s_{0}$ and perform the Borel transformation with respect to the variable $P^{2}=-p^{2}$, finally we obtain the following sum rule,

$$
\begin{aligned}
& f_{X}^{2} e^{-\frac{m_{X}^{2}}{M^{2}}}=A A \\
& A A=\int_{4 m_{s}^{2}}^{s_{0}} d t e^{-\frac{t}{M^{2}}}\left\{\frac{t^{5}}{716800 \pi^{6}}+\frac{\langle\bar{s} s\rangle\left\langle\bar{q} g_{s} \sigma G q\right\rangle+\langle\bar{q} q\rangle\left\langle\bar{s} g_{s} \sigma G s\right\rangle}{24 \pi^{2}} t\right. \\
& \left.-\frac{2\langle\bar{q} q\rangle-\langle\bar{s} s\rangle}{1920 \pi^{4}} m_{s} t^{3}+\frac{t^{3}}{15360 \pi^{4}}\left\langle\frac{\alpha_{s} G G}{\pi}\right\rangle\right\}+ \\
& \frac{\langle\bar{s} s\rangle^{2}\left\langle\bar{q} g_{s} \sigma G q\right\rangle+\langle\bar{s} s\rangle\langle\bar{q} q\rangle\left\langle\bar{s} g_{s} \sigma G s\right\rangle-2\langle\bar{s} s\rangle\langle\bar{q} q\rangle\left\langle\bar{q} g_{s} \sigma G q\right\rangle-2\langle\bar{q} q\rangle^{2}\left\langle\bar{s} g_{s} \sigma G s\right\rangle}{18} m_{s},
\end{aligned}
$$

here the $s_{0}$ is the threshold parameter. Differentiate the above sum rule with respect to the variable $\frac{1}{M^{2}}$, then eliminate the quantity $f_{X}$, we obtain

$$
\begin{aligned}
m_{X}^{2}= & B B / A A, \\
B B= & \int_{4 m_{s}^{2}}^{s_{0}} d t e^{-\frac{t}{M^{2}}}\left\{\frac{t^{6}}{716800 \pi^{6}}+\frac{\langle\bar{s} s\rangle\left\langle\bar{q} g_{s} \sigma G q\right\rangle+\langle\bar{q} q\rangle\left\langle\bar{s} g_{s} \sigma G s\right\rangle}{24 \pi^{2}} t^{2}\right. \\
& \left.-\frac{2\langle\bar{q} q\rangle-\langle\bar{s} s\rangle}{1920 \pi^{4}} m_{s} t^{4}+\frac{t^{4}}{15360 \pi^{4}}\left\langle\frac{\alpha_{s} G G}{\pi}\right\rangle\right\} .
\end{aligned}
$$

It is easy to perform the $t$ integral in Eqs.(6-7), we prefer this form for simplicity.

The input parameters are taken as $\langle\bar{s} s\rangle=(0.8 \pm 0.1)\langle\bar{q} q\rangle,\left\langle\bar{s} g_{s} \sigma G s\right\rangle=m_{0}^{2}\langle\bar{s} s\rangle$, $\left\langle\bar{q} g_{s} \sigma G q\right\rangle=m_{0}^{2}\langle\bar{q} q\rangle, m_{0}^{2}=(0.8 \pm 0.1) G e V^{2},\langle\bar{q} q\rangle=-(0.24 \pm 0.01 G e V)^{3},\left\langle\frac{\alpha_{s} G G}{\pi}\right\rangle=$ $(0.33 G e V)^{4}, m_{u}=m_{d}=0$ and $m_{s}=(0.14 \pm 0.01) G e V$. The threshold parameter is taken as $\sqrt{s_{0}}=(2.1 \pm 0.1) G e V$ to avoid possible contaminations from the high resonances and continuum states. The width of the $X(1576)$ is very broad, about $0.8 \mathrm{GeV}$, the threshold parameter $s_{0} \geq(1.6+0.4)$ can include the contribution 


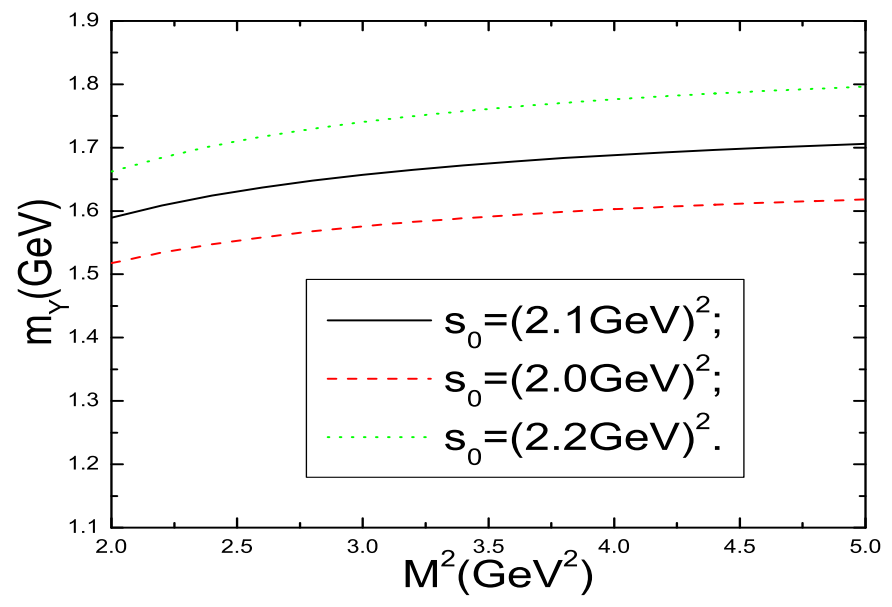

Figure 1: The $m_{X}$ with the Borel parameter $M^{2}$ for the central values of the condensates.

from the ground state vector meson. The Borel parameter $M^{2}$ is chosen to be $M^{2}=(2-5) G e V^{2}$, i.e. $M=(1.4-2.2) G e V$ and $e^{-\frac{s_{0}}{M^{2}}} \leq e^{-1}$, the contributions from the high resonances and continuum states can be suppressed, furthermore, in this region, the mass from the sum rule is rather stable, which is shown in Fig.1. For detailed discussions about the criterion of choosing the Borel parameter $M^{2}$ and threshold parameter $s_{0}$ for the multiquark states, one can consult Ref. [12. The contribution from the perturbative term is about $10 \%$, the dominating contribution comes from the term $\langle\bar{s} s\rangle\left\langle\bar{q} g_{s} \sigma G q\right\rangle+\langle\bar{q} q\rangle\left\langle\bar{s} g_{s} \sigma G s\right\rangle$, about $80 \%$, the other terms are of minor importance, we can expect the convergence of the operator product expansion.

Taking into account all the uncertainties, finally we obtain the mass of the $X(1576)$,

$$
m_{X}=(1.66 \pm 0.14) G e V
$$

Comparing with the experimental data $\left(1576_{-55-91}^{+49+98} \mathrm{MeV}\right)$ and the calculation of the quark model $(1632.854 \mathrm{MeV})$, our numerical result is reasonable, although somewhat larger, the vector meson $X(1576)$ may have some tetraquark component. In this letter, we take the scalar diquarks as the basic constituents, the instantons manifest themselves in the scalar diquark channels, the contributions of the direct instantons may account for the discrepancy [13].

The tetraquark state can mix with the molecular state if they have the same quantum numbers, the mixing can also pull the mass down. In Ref. [2, the $X(1576)$ is taken as an $S$-wave $K^{*}(892)-\bar{\kappa}$ molecular state, the large width of the $X(1576)$ is taken into account by the large width of the $\kappa$. If the negative bound energy of the molecular state is large enough, the mixing can pull the mass down significantly. 
In the tetraquark scenario, the dominant decay modes of the $X(1576)$ are $K^{+} K^{-}$, $K_{L} K_{S}$ and $\phi \pi^{0}$ which are OZI-allowed, not the $\pi^{+} \pi^{-}$which is OZI-forbidden [3. In the $K^{*}(892)-\bar{\kappa}$ molecular scenario, the decay mode $\pi^{+} \pi^{-}$has much larger branching ratio than the decay mode $K^{+} K^{-}$2. Whether or not there exist the resonant structure in the decay $J / \psi \rightarrow \pi^{+} \pi^{-} \pi^{0}$ is of great importance for understanding the structure of the $X(1576)$.

In this letter, we take the point of view that the $X(1576)$ be tetraquark state which consists of a scalar-diquark and an anti-scalar-diquark in relative $P$-wave, and calculate its mass in the framework of the QCD sum rules approach. The numerical value of the mass is consistent with the experimental data, there may be some tetraquark component in the vector meson $X(1576)$.

\section{Acknowledgment}

This work is supported by National Natural Science Foundation, Grant Number 10405009, and Key Program Foundation of NCEPU.

\section{References}

[1] M. Ablikim, et al. , hep-ex/0606047.

[2] F. K. Guo, P. N. Shen, hep-ph/0606273.

[3] G. J. Ding, M. L. Yan, hep-ph/0607253.

[4] M. Karliner, H. J. Lipkin, hep-ph/0607093.

[5] M. A. Shifman, A. I. Vainshtein and V. I. Zakharov, Nucl. Phys. B147 (1979) $385,448$.

[6] A. De Rujula, H. Georgi and S. L. Glashow, Phys. Rev. D12 (1975) 147 ; T. DeGrand, R. L. Jaffe, K. Johnson and J. E. Kiskis, Phys. Rev. D12 (1975) 2060; T. Schafer and E. V. Shuryak, Rev. Mod. Phys. 70 (1998) 323.

[7] Z. G. Wang, W. M. Yang, S. L. Wan , J. Phys. G31 (2005) 971; Z. G. Wang, W. M. Yang, Eur. Phys. J. C42 (2005) 89; T. V. Brito, F. S. Navarra, M. Nielsen and M. E. Bracco, Phys. Lett. B608 (2005) 69.

[8] F. E. Close and N. A. Tornqvist, J. Phys. G28 (2002) R249; R. L. Jaffe, Phys. Rept. 409 (2005) 1; C. Amsler and N. A. Tornqvist, Phys. Rept. 389 (2004) 61 ; and references therein.

[9] N. Isgur, G. Karl, Phys. Rev. D18 (1978) 4187.

[10] M. Hess, F. Karsch, E. Laermann, I. Wetzorke, Phys. Rev. D58 (1998) 111502. 
[11] Z. G. Wang, S. L. Wan, W. M. Yang, hep-ph/0506035.

[12] Z. G. Wang, S. L. Wan, Nucl. Phys. A778 (2006) 22; Z. G. Wang, S. L. Wan, hep-ph/0601105.

[13] H. Forkel, M. K. Banerjee, Phys. Rev. Lett. 71 (1993) 484; H. Forkel, Phys. Rev. D71 (2005) 054008. 